\title{
The Effects of Bioavailable Curcumin (Cureit) on Delayed Onset Muscle Soreness Induced By Eccentric Continuous Exercise: A Randomized, Placebo-Controlled, Double-Blind Clinical Study
}

\author{
Augustine Amalraj, ${ }^{1}$ Chandradhara Divya, ${ }^{2}$ and Sreeraj Gopi ${ }^{1}$ \\ ${ }^{1} R \& D$ Centre, Aurea Biolabs Private Limited, Kolenchery, Cochin, Kerala, India. \\ ${ }^{2}$ BioAgile Therapeutics Private Limited, Bangalore, Karnataka, India.
}

\begin{abstract}
Delayed onset muscle soreness (DOMS) is a multifactorial progression related to muscle pain, swelling, stiffness, tenderness, altered joint kinematics, muscle fiber disruption, decreased strength and power, and acute tissue damage. Curcumin, a natural phytonutrient, could manage DOMS induced by eccentric continuous exercise due to its wide range of biological activities. This study was a randomized, placebo-controlled, double-blind clinical study to assess the efficacy of Cureit $^{\mathrm{TM}}$ - a bioavailable form of curcumin that may decrease damage from inflammation and oxidative stress associated with severe muscle damage induced by continuous eccentric exercise. The results of the study showed that oral consumption of Cureit significantly reduced DOMS, slightly reduced creatinine kinase concentrations, and slightly increased $\mathrm{VO}_{2}$ max value compared with placebo, and found safe for administration. The consumption of Cureit led to improved recovery and reduction of DOMS without any side effects due to the enhancement of bioavailable form of curcumin.
\end{abstract}

KEYWORDS: • curcumin • delayed onset muscle soreness $\bullet$ muscle damage $\bullet$ eccentric muscle injury

\section{INTRODUCTION}

D ELAYED ONSET MUSCLE SORENESS (DOMS) is a multifactorial process associated with muscle pain, stiffness, swelling, tenderness, altered joint kinematics, muscle fiber disruption, acute tissue damage, and decreased strength and power. ${ }^{1}$ DOMS, which presents as tenderness and muscle pain, characteristically ensues afterward a spirited exercise or engaging in unfamiliar exercise, which are also related to sarcomere disruption and the ensuing inflammatory response. ${ }^{2}$ Although the precise mechanisms responsible for these signs and symptoms are not completely understood, inflammation and reactive oxygen species are presumably the core cause of DOMS and other possible causes include disruption of the excitation/contraction coupling process, disorganization of myofilaments, z-line widening and streaming, and a resistant response that creates a gathering of mononuclear cells. ${ }^{3}$ At present, there are multiple strategies that have been treated to attenuate DOMS such as hyperbaric oxygen, anti-inflammatory medication, massage, cryotherapy, homeopathy, stretching, electrotherapeutic modalities, ultrasound, rest, and light exercise. ${ }^{4,5}$

Nutritional interventions with antioxidant and antiinflammatory properties are relevant to reduce DOMS,

Manuscript received 3 July 2019. Revision accepted 23 December 2019.

Address correspondence to: Sreeraj Gopi, PhD, R\&D, Centre, Aurea Biolabs Private Limited, Kolenchery, Cochin 682 311, Kerala, India, E-mail: sreeraj.gopi@ plantlipids.com; sreerajgopi@yahoo.com specifically the consumption of biologically active components. ${ }^{6,7}$ By means of a natural alternative therapy to manage DOMS, curcumin has in recent years become recognized as an important natural intervention due to its extensive range of biological activities, including antimicrobial, anticancer, antiinflammatory, antitumor, antioxidant, radioprotective, cardioprotective, and neuroprotective properties, ${ }^{8}$ even though the biological applications of curcumin have been restricted by its very low solubility, poor absorption, bioavailability, quick elimination, and metabolic changes. The researchers at Aurea Biolabs developed an innovative bioavailable curcumin $\left(\right.$ Cureit $\left.^{\mathrm{TM}}\right)$ based on the recreation of the complete natural turmeric matrix with active curcumin using polar/nonpolarsandwich (PNS) technology. ${ }^{9}$ Cureit already has demonstrated efficacy from different studies such as antioxidant, antineoplastic, elastase inhibition, antiaging, and antirheumatoid arthritis efficacies. ${ }^{10-15}$

Acceptance and delivery of curcumin in body tissues are perceptibly important for its biological activities. The bioavailability of curcuminoids was assessed in a detailed pharmacokinetic study of Cureit, which clearly demonstrated the synergism of curcuminoids with other bioactive molecules of turmeric, and showed that the PNS technology made a positive impact on generating high concentration of free curcuminoids in the blood plasma, which could play a significant role in treating pain and DOMS. ${ }^{16}$ Moreover, curcuminoids have well-documented anti-inflammatory, antioxidant, and anticarcinogenic properties. ${ }^{8}$ The curcuminoids exhibit pleiotropic actions, interacting with several 
molecular targets involved in inflammation, including tumor necrosis factor- $\alpha$, nuclear factor-jB, interleukin (IL)- $1 \beta$, IL-6, IL-8, and cyclooxygenase-2. ${ }^{17,18}$ These pleiotropic actions of Cureit containing curcuminoids could play an important role in decreasing the severity of the muscular pain following DOMS, which suggests that Cureit could be an ideal candidate for treating DOMS.

Based on the earlier studies and data, Cureit could have advantageous effects on muscle damage, soreness, and recovery. Cureit could have a positive impact on DOMS, excessive stress of high-intensity exercise, and precipitating biochemical and hormonal responses. Therefore, curcumin with all of the other bioactive molecules of turmeric would be a prospective approach to improve exercise recovery for persons to join training programs or to undergo progressions in the exercise workload. The aim of this study was to assess the efficacy of Cureit for treating subjects with DOMS due to eccentric muscle activity, to monitor the adverse events, and to ensure the general well-being and safety of subjects.

\section{MATERIALS AND METHODS}

\section{Ethical approval}

The study protocol was approved by the Institutional Ethics Committee (IEC) from Rajalakshmi Hospital, Bengaluru, Karnataka, India. The protocol complied with recommendations of the 18th World Health Congress (Helsinki, 1964) and all applicable amendments. The protocol also complied with the Good Clinical Practice (GCP) and Helsinki Declaration standards, as well as per Indian GCP and Schedule Y (amended version 2005) and Indian Council of Medical Research codes. Written informed consent was obtained from each subject. The study was registered with Clinical Trials Registry India (clinicaltrials.gov) (CTRI/2018/05/014174).

\section{Subjects}

A series of screening evaluations, including demography, physical examination, vital signs, hematology, biochemistry, marker analysis of creatine kinase (CK), and $\mathrm{VO}_{2}$ max (treadmill), were performed after obtaining informed consent to determine whether prospective study subjects meet the section criteria for the study. A total of 33 subjects were screened to enroll 30 subjects (age $36 \pm 11$ years; male [ $n=12]$ and female $[n=18]$ ) who were randomized into two treatment groups. The flowchart of the allocation of subjects is described in Figure 1. The demographic data, containing weight, height, and body mass index (BMI), and vital signs containing pulse, systolic blood pressure, diastolic blood pressure, and respiratory rate, were measured and determined (Table 1) in both screening and end of the study period.

\section{Supplementation}

The bioavailable form of curcumin - Cureit, was supplied by Aurea Biolabs (P) Ltd., Cochin, Kerala, India. The placebo was visually similar to Cureit but contained a food- grade starch. The subjects received a single oral $500 \mathrm{mg}$ dosage of one of the products in capsule form.

\section{Study design}

The present study was a randomized, placebo-controlled, double-blind clinical study to test whether Cureit-the bioavailable form of curcumin, could decrease damage from oxidative stress and inflammation related to severe muscle damage induced by eccentric continuous exercise. The study design is schematically represented in Figure 2.

\section{Inclusion and exclusion criteria}

The inclusion criteria included subjects who were 20 years and older, healthy, moderately active that is regular aerobic exercise for no less than $4 \mathrm{~h}$ per week, no known musculoskeletal pathology, and nonsmokers. The subjects should have a maximal oxygen consumption $\left(\mathrm{VO}_{2} \max \right)$ of no less than $35 \mathrm{~mL} / \mathrm{kg}$, as measured by the maximal treadmill exercise test, and who were willing to sign an informed consent and adhere to all protocol requirements.

The exclusion criteria included subjects who had taken anti-inflammatory/analgesic/antioxidant drugs in the prior month, had unusual liver or renal function tests, laboratory findings indicative of an active infectious or inflammatory process and occurrence of any known illnesses, pregnancy or breastfeeding, current alcoholism or drug abuse, allergies to one of the component ingredients in the trail, active prescription, and involvement in additional clinical studies less than 3 months formerly.

\section{Randomization and blinding}

As the subjects entered the study, randomization number and associated treatment were assigned sequentially by the study statistician; once EC approval was received, the statistician blinded the samples by removing the product label and adhered a blinded label according to the randomization procedures against unique codes. This randomization schedule was made available to the personnel responsible for packaging/labeling/blinding of the investigational product. The same list was also provided for the analysis/reporting database for analysis and completion of study (after data lock process).

The blinding was done via a computer-generated process by the team statistician; investigational product numbers were preprinted as stickers and were affixed on each kit, which was the only identity to separate one kit from the other. The investigator assigned treatment kits to the enrolled subjects as per the schedule. The treatment kit included the blinded product. Fifteen subjects were randomized into each Cureit group and placebo group. Each subject consumed one capsule daily during visits 2,3 , and 4 .

\section{Eccentric muscle injury protocol}

DOMS and associated muscle damage were induced by downhill treadmill running. A downhill running protocol as described by Nurenberg et al. ${ }^{19}$ and Malm et al. ${ }^{20}$ was used to induce the eccentric muscle injury with suitable modifications. 


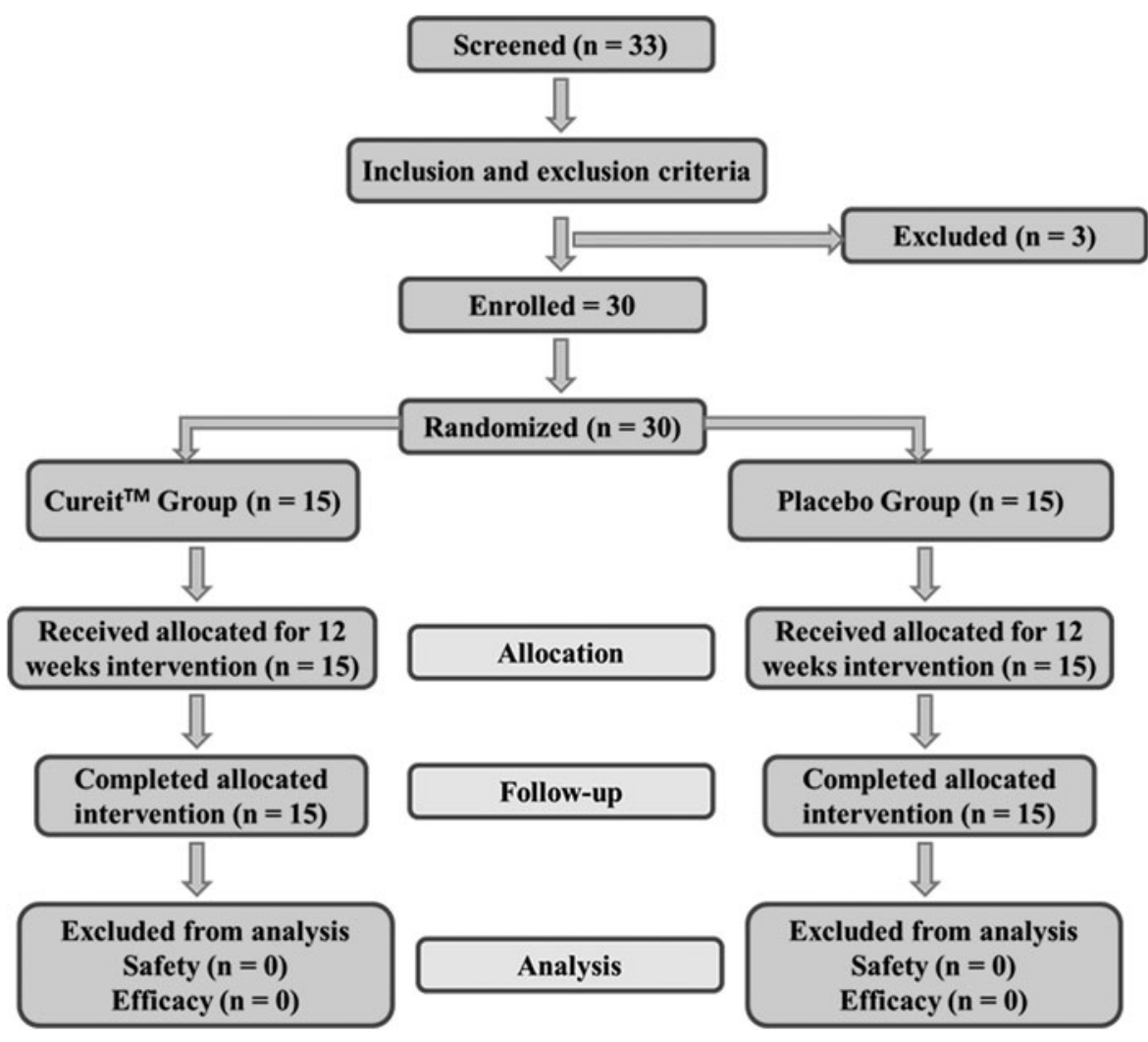

FIG. 1. Flowchart of the subjects.

Subjects chose a speed for the 10-min warm-up and then ran downhill with a $10 \%$ treadmill grade at a constant speed for $45 \mathrm{~min}$. Initially, the treadmill speed was set at $6 \mathrm{~km} / \mathrm{h}$ and increased by $1 \mathrm{~km} / \mathrm{h}$ each min until reaching the maximum maintainable effort such as muscle tiredness or maintenance or weakening in $\mathrm{VO}_{2}$ max. The treadmill grade was fixed at 3\% and was maintained during the entire test. Subjects performed 10-min exercise sessions during the screening periods to familiarize themselves with the exercise protocol and to analyze more susceptible muscle fibers, so that could attain similar fiber arrangement and normalize the starting point level in all subjects. All the subjects received an oral nutritional supplement containing 2-4 g of protein and 25-30 g of carbohydrate, $1 \mathrm{~h}$ before, and $500 \mathrm{~mL}$ of drinking water for $30 \mathrm{~min}$ before the start of the eccentric muscle injury test. The subjects were permitted to drink water during the study. The $\mathrm{VO}_{2}$ max was recorded for each subject at the screening visit (day-7) and at the end of the study (day 4). Pain intensity was assessed in the subjects for $48 \mathrm{~h}$ in every visit after downhill running using a visual analog scale (VAS). Subjects were questioned to show the location of pain on a drawing signifying the lower limbs, and to rank the pain intensity on a $0-4$-point VAS, where $0=$ no pain and $4=$ disabling pain when descending or climbing stairs.

\section{Blood sampling and laboratory analysis}

One week before the study, regular laboratory analyses such as complete blood count, C-reactive protein, erythrocyte sedimentation rate, alanine aminotransferase, aspartate aminotransferase, cholesterol, urea, alkaline phosphatase, creatinine, triglycerides, total high-density lipoproteincholesterol, and low-density lipoprotein-cholesterol were executed to evaluate the suitability of the subjects and the same was repeated at the end of the study on day 4 of the treatment. Blood sampling was collected instantly earlier the downhill running test at the screening day (visit 1) and end of the study (day 4) after the downhill running test for the measurement of CK, which is utilized as an indicator of muscle damage, hematology, biochemistry, and other vital signs.

\section{Outcome measures}

In this study, the efficacy of the Cureit was evaluated using the following outcomes: improvement in the stabilization of muscles or reduction in the degree of muscle fatigue at $\mathrm{VO}_{2}$ max (increase in value), decrease in levels of CK by the oxidative stress and inflammatory marker test, reduction in pain, which was identified using VAS, and improvement with Cureit compared with the placebo. Also, baseline values were compared with post-treatment (postconsumption of the Cureit or placebo for 4 days). In addition, Global Evaluation by Patient Questionnaire was also evaluated. The safety of Cureit was also analyzed by any incidence of adverse events.

\section{Statistical analysis}

Subjects were randomly assigned to each arm equally using a blocked randomization method. Summary statistics was applied to the related data. Analysis for all parameters 
Table 1. Subject Characteristics

\begin{tabular}{|c|c|c|c|c|}
\hline \multirow[b]{2}{*}{ Parameter/statistics } & \multicolumn{2}{|c|}{ Cureit $^{\mathrm{TM}}(\mathrm{n}=15)$} & \multicolumn{2}{|c|}{ Placebo $(\mathrm{n}=15)$} \\
\hline & Screening & Visit 5 & Screening & Visit 5 \\
\hline \multicolumn{5}{|l|}{ Height $(\mathrm{cm})$} \\
\hline Mean \pm SD & $159.9 \pm 4.9$ & $159.9 \pm 4.9$ & $161.7 \pm 4.6$ & $161.7 \pm 4.6$ \\
\hline Median & 158.6 & 158.6 & 163.5 & 163.5 \\
\hline Min., Max. & $153.9,169.4$ & $153.9,169.4$ & $153.8,167.2$ & $153.8,167.2$ \\
\hline SEM & 1.2681 & 1.2607 & 1.1755 & 1.1755 \\
\hline$\% \mathrm{CV}$ & 3.07 & 3.05 & 2.81 & 2.81 \\
\hline \multicolumn{5}{|l|}{ Weight (kg) } \\
\hline Mean \pm SD & $60.3 \pm 3.9$ & $60.3 \pm 3.8$ & $60.3 \pm 4.3$ & $60.4 \pm 4.3$ \\
\hline Median & 60.3 & 60.2 & 59.7 & 60.0 \\
\hline Min., Max. & $53.6,67.0$ & $53.9,67.0$ & $54.3,72.6$ & $54.2,72.9$ \\
\hline SEM & 0.9970 & 0.9893 & 1.0996 & 1.1184 \\
\hline$\% \mathrm{CV}$ & 6.40 & 6.35 & 7.07 & 7.17 \\
\hline \multicolumn{5}{|l|}{ BMI $\left(\mathrm{kg} / \mathrm{m}^{2}\right)$} \\
\hline Mean \pm SD & $23.60 \pm 1.13$ & $23.59 \pm 1.10$ & $23.06 \pm 1.67$ & $23.08 \pm 1.68$ \\
\hline Median & 23.83 & 23.85 & 22.96 & 22.92 \\
\hline Min., Max. & $21.35,25.70$ & $21.39,25.66$ & $21.00,26.79$ & $21.00,26.91$ \\
\hline SEM & 0.2924 & 0.2834 & 0.4305 & 0.4350 \\
\hline$\% \mathrm{CV}$ & 4.80 & 4.65 & 7.23 & 7.30 \\
\hline \multicolumn{5}{|c|}{ Pulse (beats per minutes) } \\
\hline Mean \pm SD & $76 \pm 3$ & $75 \pm 2$ & $75 \pm 4$ & $75 \pm 3$ \\
\hline Median & 78 & 74 & 76 & 76 \\
\hline Min., Max. & 70,80 & 70,78 & 68,80 & 70,80 \\
\hline SEM & 0.8047 & 0.6110 & 0.9085 & 0.6705 \\
\hline$\% \mathrm{CV}$ & 4 & 3 & 5 & 3 \\
\hline \multicolumn{5}{|c|}{ Systolic blood pressure (mmHg) } \\
\hline Mean \pm SD & $124 \pm 4$ & $123 \pm 2$ & $123 \pm 5$ & $124 \pm 4$ \\
\hline Median & 124 & 122 & 124 & 124 \\
\hline Min., Max. & 118,128 & 120,128 & 110,128 & 120,130 \\
\hline SEM & 0.9801 & 0.6110 & 1.2293 & 0.9043 \\
\hline$\% \mathrm{CV}$ & 3 & 2 & 4 & 3 \\
\hline \multicolumn{5}{|c|}{ Diastolic blood pressure $(\mathrm{mmHg})$} \\
\hline Mean \pm SD & $74 \pm 3$ & $73 \pm 2$ & $74 \pm 3$ & $74 \pm 2$ \\
\hline Median & 76 & 74 & 74 & 74 \\
\hline Min., Max. & 70,78 & 70,76 & 68,80 & 70,80 \\
\hline SEM & 0.7091 & 0.6005 & 0.7920 & 0.6434 \\
\hline$\% \mathrm{CV}$ & 4 & 3 & 4 & 3 \\
\hline \multicolumn{5}{|c|}{ Respiration rate (breaths per minutes) } \\
\hline Mean \pm SD & $20 \pm 1$ & $20 \pm 1$ & $20 \pm 1$ & $20 \pm 1$ \\
\hline Median & 20 & 20 & 20 & 20 \\
\hline Min., Max. & 18,22 & 18,20 & 18,22 & 18,22 \\
\hline SEM & 0.3838 & 0.1817 & 0.3634 & 0.3305 \\
\hline$\% \mathrm{CV}$ & 8 & 4 & 7 & 6 \\
\hline
\end{tabular}

BMI, body mass index; CV, coefficient of variation; SD, standard deviation; SEM, standard error of the mean.

was performed for the primary variables using baseline as covariates by analysis of covariance (ANCOVA). Also, analysis was performed comparing baseline values with post-treatment values for both Cureit and placebo using analysis of variance (ANOVA) with the level of significance $(\alpha)$ as $P<.05$.

\section{RESULTS}

In this study, the characteristics of the subjects such as height, weight, BMI, pulse, systolic blood pressure, diastolic blood pressure, and respiration rate are given in Table 1 and there are no significant changes between the screening and end of the study (visit 5). There were no serious adverse events during the study period. In this study, the efficacy of Cureit was evaluated by different functional measures, including $\mathrm{VO}_{2}$ max test, levels of $\mathrm{CK}$, and VAS score, and the results are shown in Figure 3. The CK was determined to be $129.93 \mathrm{U} / \mathrm{L}$ for the Cureit and 112.87 U/L in the placebo group at the screening stage (visit 1) following the downhill running, signifying that muscle damage had probably occurred. However, the CK was lower (109.73 U/L) at end of the study (visit 5) in the Cureit group and the CK was higher $(125.40 \mathrm{U} / \mathrm{L})$ at end of the study in the placebo group 


\section{Potential Subjects}

$\sqrt{5}$

\section{Visit 1 - Screening/Baseline/Randomization (-7 Day)}

Informed consent, demography, physical examination, medical history, urine pregnancy test, vital signs, inclusion/ exclusion criteria, randomization, haematology, biochemistry and marker analysis of $\mathrm{CK}$, muscle fatigue or stabilization/decline in $\mathrm{VO}_{2}$ max, pain intensity test and global evaluation by patient questionnaire.

Visit 2 - (Day 1)

Physical examination, urine pregnancy test, vital signs, investigational product (IP) dispensing and instructions, IP complaince, adverse events/serious adverse events assessment and concomitant medication details.

Visit 3 - (Day 2)

Physical examination, urine pregnancy test, vital signs, IP dispensing and instructions, IP compliance, adverse events/serious adverse events assessment and concomitant medication details.

\section{II}

\section{Visit 4 - (Day 3)}

Physical examination, urine pregnancy test, vital signs, IP dispensing and instructions, IP compliance, adverse events/serious adverse events assessment and concomitant medication details.

\section{Visit 5 - (Day 4)}

Physical examination, urine pregnancy test, vital signs, IP dispensing and instructions, IP compliance, adverse events/serious adverse events assessment and concomitant medication details, haematology, biochemistry and marker analysis of CK, muscle fatigue or stabilization/decline in $\mathrm{VO}_{2}$ max, pain intensity test and global evaluation by patient and investigator questionnaire.

Study Termination

/Completion Details

FIG. 2. Experimental design. CK, creatine kinase.

(Table 2). Statistical analysis (ANCOVA) showed that there was no significant difference $(P=.1873)$ observed in the $\mathrm{CK}$ levels of Cureit and placebo; furthermore, ANOVA of the CK levels between screening (visit 1) and end of the study (visit 5) showed no significant difference for either the Cureit $(P=.4566)$ or placebo $(P=.3617)$ groups.

The $\mathrm{VO}_{2}$ max is the maximum amount of oxygen the body can use, and it directly correlated with fitness capacity. In this study, whether the Cureit administration helped to improve $\mathrm{VO}_{2}$ max value was assessed. The $\mathrm{VO}_{2} \max$ value was registered 38.87 for the Cureit and 40.24 in the placebo group at the screening stage (visit 1) subsequent to the downhill running, but the CK was slightly increased (39.80) at end of the study (visit 5) in the Cureit group, and instead, CK was slightly decreased (38.97) at end of the study in the placebo group (Table 2). Statistical analysis (ANCOVA) showed significant differences $(P=.0009)$ observed in the
$\mathrm{VO}_{2}$ max value of Cureit and placebo; nevertheless, ANOVA of $\mathrm{VO}_{2}$ max value between the screening and end of the study showed no significant difference for Cureit $(P=.2846)$ and placebo $(P=.2517)$.

The pain effect measures revealed a high VAS score at screening and postexercise in both groups, signifying that the exercise loading protocol was effective at creating DOMS. DOMS was measured in both Cureit- and placebotreated subjects using the pain VAS, DOMS typically peaks 48 to $72 \mathrm{~h}$ after exercise, as the body surely exerts a lot of energy in the process to repair muscle fibers that were uncertain during exercise. There are changing grades of pain dependent on how much destruction has been done and other influences such as genetics and hydration. This study assessed the impact of administration of Cureit to reduce DOMS. The pain VAS score significantly decreased from 2.90 to 1.17 in the subjects treated with Cureit from the 
a
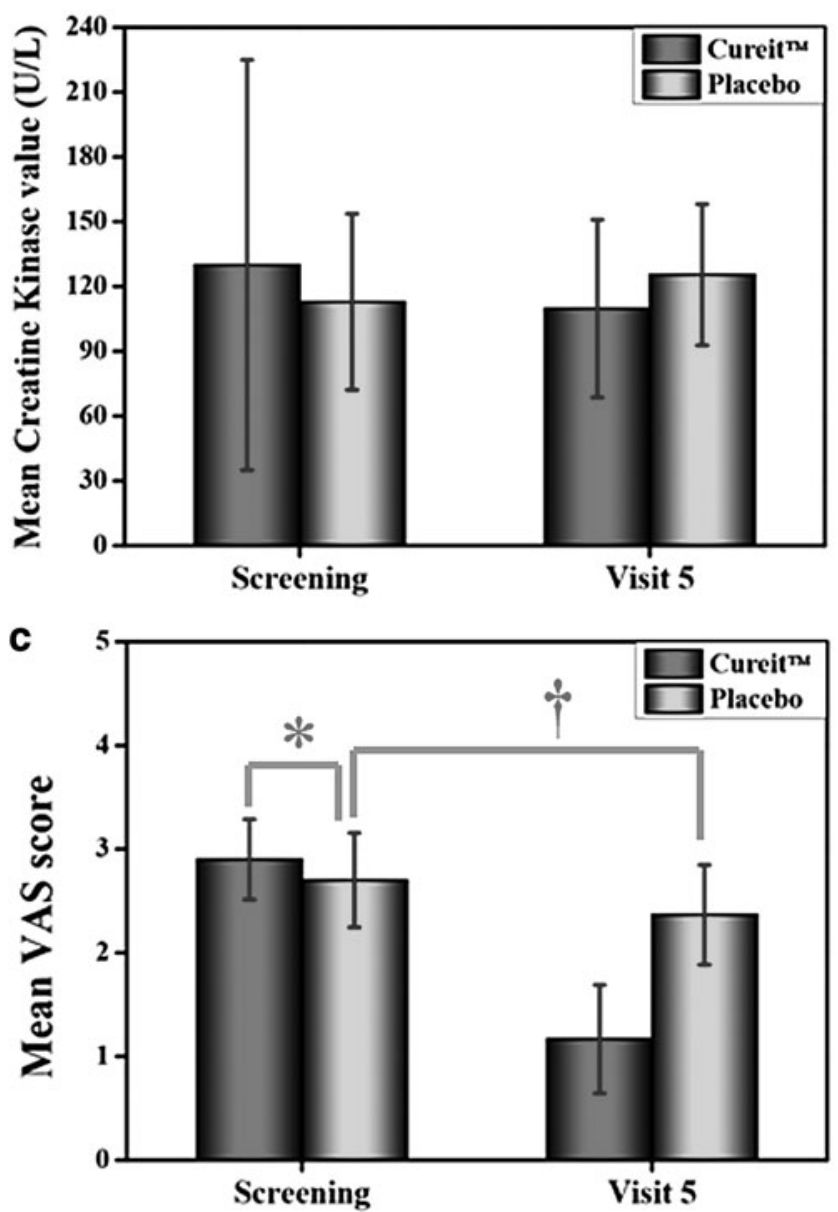

b
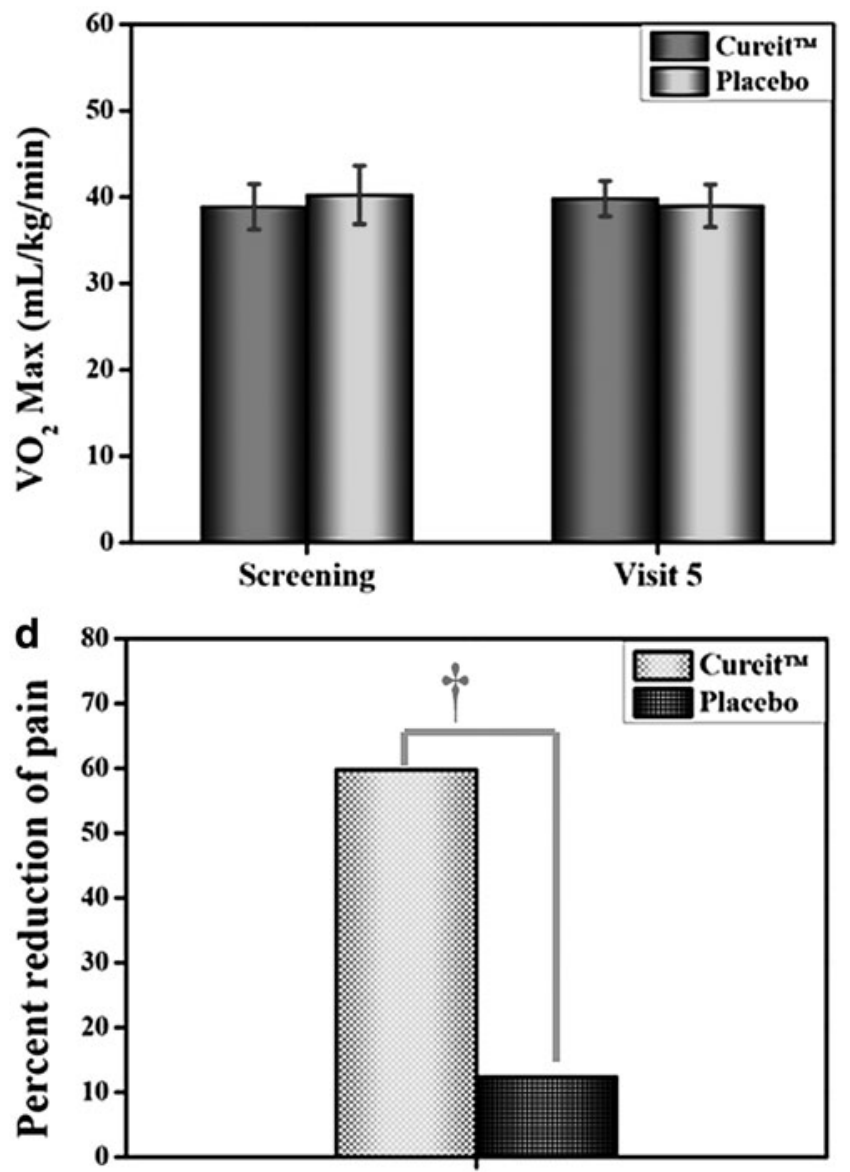

FIG. 3. Muscle soreness assessed in the screening and end of the study (visit 5) between the Cureit ${ }^{\mathrm{TM}}$ and placebo groups using (a) creatine kinase, (b) $\mathrm{VO}_{2}$ max test, (c) VAS score, and (d) percent reduction of pain (mean $\pm \mathrm{SD}$ ). *Significant difference between screening and end of the study at $P<.05$; ${ }^{\dagger}$ significant difference between the Cureit and placebo groups at $P<.05$. SD, standard deviation; VAS, visual analog scale.

screening to end of the study period; in contrast, the pain VAS score slightly decreased from 2.70 to 2.37 in the placebo group between the screening and end of the study period (Table 2). Statistical analysis (ANCOVA) showed significant differences $(P<.001)$ in the pain score of the Cureit and placebo groups; moreover, the ANOVA of pain score between the screening and end of the study showed a significant difference for the Cureit $(P<.0001)$, whereas there were no significant differences in the placebo group $(P=.061)$.

Overall results were also confirmed by the global evaluation using the patient questionnaire study (Table 2), which indicated generally no or less pain with Cureit-treated subjects when comparing the screening day to the end of the study, whereas the placebo-treated subjects felt almost a similar degree of pain during the screening and end of the study. The results of the present study indicated that the bioavailable form of curcumin - Cureit, in a complete natural turmeric matrix could provide significant improvements against DOMS when compared with placebo.

\section{DISCUSSION}

Curcumin has multiple biological effects that may include antimicrobial, antioxidant, anticancer, anti-inflammatory, radioprotective, neuroprotective, and cardioprotective effects that are supported by many clinical trials, and acts through a number of signaling molecules by binding them directly or indirectly by different bonds such as covalent, noncovalent, hydrogen, and hydrophobic bonding. Furthermore, curcumin could directly interact with different signaling molecules, particularly inflammatory molecules. ${ }^{8,21}$ To the best of our knowledge, very few studies have investigated the effect of curcumin on DOMS, because of its poor solubility, bioavailability, and rapid elimination. In this study, a highly bioavailable form of curcumin - Cureit, was used, which is developed based on the restructuring of the complete natural turmeric matrix by the patented PNS technology. This could be an excellent strategy to overcome all the limitations of curcumin and improve its efficacy for DOMS as well as pain and inflammatory conditions due to 
Table 2. Effect of Cureit on Different Functional Measures of Muscle Damage and Fatigue Following Eccentric Exercise

\begin{tabular}{|c|c|c|c|c|c|c|c|}
\hline \multirow[b]{2}{*}{ Parameter/statistics } & \multicolumn{3}{|c|}{ Cureit $(\mathrm{n}=15)$} & & \multicolumn{3}{|c|}{ Placebo $(\mathrm{n}=15)$} \\
\hline & Screening & & Visit 5 & & Screening & & Visit 5 \\
\hline \multicolumn{8}{|l|}{ CK values (U/L) } \\
\hline Mean \pm SD & $129.93 \pm 94.94$ & & $109.73 \pm 41.16$ & & $112.87 \pm 40.77$ & & $125.40 \pm 32.66$ \\
\hline Median & 99.00 & & 105.00 & & 101.00 & & 116.00 \\
\hline Min., Max. & $52.00,393.00$ & & $43.00,184.00$ & & $59.00,194.00$ & & $79.00,187.00$ \\
\hline SEM & 24.51 & & 10.63 & & 10.53 & & 8.43 \\
\hline$\% \mathrm{CV}$ & 73.07 & & 37.51 & & 36.12 & & 26.05 \\
\hline $\begin{array}{l}P \text { value (screening vs. visit } 5 \text { ) } \\
P \text { value (Cureit vs. placebo) }\end{array}$ & & .4566 & & .1873 & & .3617 & \\
\hline \multicolumn{8}{|l|}{ Treadmill/ $/ \mathrm{VO}_{2}$ max test $(\mathrm{mL} / \mathrm{kg} / \mathrm{min})$} \\
\hline Mean \pm SD & $38.87 \pm 2.64$ & & $39.80 \pm 2.04$ & & $40.24 \pm 3.38$ & & $38.97 \pm 2.47$ \\
\hline Median & 40.35 & & 41.05 & & 40.35 & & 38.96 \\
\hline Min., Max. & $35.41,41.65$ & & $36.39,42.09$ & & $35.95,45.95$ & & $35.52,44.28$ \\
\hline SEM & 0.68 & & 0.53 & & 0.87 & & 0.64 \\
\hline$\% \mathrm{CV}$ & 6.79 & & 5.12 & & 8.40 & & 6.33 \\
\hline$P$ value (screening vs. visit 5 ) & & .2846 & & & & .2517 & \\
\hline$P$ value (Cureit vs. placebo) & & & & .0009 & & & \\
\hline \multicolumn{8}{|l|}{ Pain score } \\
\hline Mean \pm SD & $2.90 \pm 0.39$ & & $1.17 \pm 0.52$ & & $2.70 \pm 0.46$ & & $2.37 \pm 0.48$ \\
\hline Median & 3.00 & & 1.00 & & 3.00 & & 2.00 \\
\hline Min., Max. & $2.00,3.50$ & & $1.00,3.00$ & & $2.00,3.00$ & & $2.00,3.00$ \\
\hline SEM & 0.100 & & 0.135 & & 0.118 & & 0.124 \\
\hline$\% \mathrm{CV}$ & 13.36 & & 44.85 & & 16.86 & & 20.31 \\
\hline$P$ value (screening vs. visit 5 ) & & $<.0001$ & & & & .0613 & \\
\hline$P$ value (Cureit vs. placebo) & & & & $<.0001$ & & & \\
\hline \multicolumn{8}{|c|}{ Global evaluation by patient questionnaire } \\
\hline Mean \pm SD & $2.67 \pm 0.49$ & & $1.13 \pm 0.35$ & & $2.73 \pm 0.46$ & & $2.40 \pm 0.51$ \\
\hline Median & 3.00 & & 1.00 & & 3.00 & & 2.00 \\
\hline Min., Max. & $2.00,3.00$ & & $1.00,2.00$ & & $2.00,3.00$ & & $2.00,3.00$ \\
\hline SEM & 0.126 & & 0.091 & & 0.118 & & 0.131 \\
\hline$\% \mathrm{CV}$ & 18.30 & & 31.05 & & 16.75 & & 21.13 \\
\hline$P$ value (screening vs. visit 5 ) & & $<.0001$ & & & & .0692 & \\
\hline$P$ value (Cureit vs. placebo) & & & & $<.0001$ & & & \\
\hline
\end{tabular}

Analysis for all parameters was performed using baseline as covariates using ANCOVA and comparing baseline values with post-treatment values for both Cureit and placebo using ANOVA with the level of significance $(\alpha)$ as $P<.05$.

ANCOVA, analysis of covariance; ANOVA, analysis of variance; $\mathrm{CK}$, creatine kinase.

the availability of other active constituents of turmeric such as bisacurone, turmerone, elemene, germacrone, protein, carbohydrates, and dietary fibers. ${ }^{9,15}$ The initial aim of this study was to evaluate the effectiveness of Cureit in subjects with DOMS due to eccentric muscle activity. The key outcomes revealed that the consumption of Cureit could have the potential to attenuate inflammatory markers and relieve muscle soreness. From the observations of this study, 4 days of Cureit supplementation lead up to a downhill running exercise, reduced the instant DOMS and inflammatory reaction to destruction exercise when linked to the placebo treatment.

The presence of the CK in the blood plasma is generally considered an indirect marker of muscle injury. ${ }^{22}$ The CK levels were decreased (15.55\%) in the subjects treated with Cureit, but instead, the CK levels were increased (11.10\%) in the subjects treated with placebo, which indicated that Cureit can attenuate DOMS-related muscle damage induced by eccentric exercise and enhance the recovery of DOMS. The anti-inflammatory and antioxidant effects of curcumin are well acknowledged and various clinical studies have reported significant decreases in the levels of oxidative stress and the inflammatory response. ${ }^{8,21}$ Cureit caused significant decreases in DOMS-associated pain symptoms, which were connected with lesser blood CK due to the bioavailable form of curcumin. ${ }^{16,23}$

The data from Table 2 suggest that the $\mathrm{VO}_{2}$ max value was slightly increased $(2.42 \%)$ in the subjects treated with Cureit, which reveals that consumption of Cureit exerts a positive impact on muscle function recovery; on the contrary, $\mathrm{VO}_{2}$ max value was found to be slightly decreased $(3.14 \%)$ in subjects treated with placebo. The pain VAS score was significantly decreased (59.77\%) in subjects treated with Cureit. On the contrary, the pain score was found to be slightly decreased by $12.34 \%$ in subjects treated with placebo. A significant reduction in the VAS score was obtained using a dietary supplement containing curcumin, phytosterols from unsaponifiable avocado, soya bean oil, proteolytic enzymes, resveratrol, and vitamin $\mathrm{C}$ in a randomized, double-blind, placebo-control, crossover pilot study for the reduction of 
DOMS after eccentric exercise. ${ }^{24}$ A hydrogenated bioavailable curcumin formulation also acts as an anti-inflammatory and analgesic therapy for the management of rheumatoid arthritis by decreasing the inflammation, which was confirmed by the significant reduction of VAS. ${ }^{25}$ Bannuru et al. reviewed and explained that various curcuminoid preparations had better efficacy than placebo for pain relief and functional movement, which was confirmed by VAS measurements with safety outcomes. ${ }^{26}$ These data suggested that consuming bioavailable curcumin-Cureit, could attenuate the muscle damage by eccentric muscle activity and facilitating muscle function recovery when compared with placebo.

Other dietary supplements with anti-inflammatory and antioxidant properties have also been revealed to reduce muscle soreness and damage markers. A green tea extract supplement reduced the markers of muscle damage after exercise, but did not reduce the sensation of DOMS, which suggested that green tea extract supplement has positive effects on muscle recovery after vigorous exercise. ${ }^{7}$ Likewise, consumption of theaflavin-enriched black tea extracts managed to improve suppression of and recovery from oxidative stress and DOMS response to severe anaerobic damage. ${ }^{27}$ A supplement containing marine oil lipid rich in n-3-long-chain polyunsaturated fatty acids derivative of the New Zealand green lipped mussel is used as a healing agent to attenuate muscle damage and inflammation subsequent to muscle destructive exercise. ${ }^{4}$ In addition, a lipid extract of the New Zealand green lipped mussel, which is rich in docosahexaenoic acid, eicosapentaenoic acid, eicosatetraenoic acid, octadecatrienoic acid, polar lipids, sterol esters, and carotenoids, is known to be a potent natural antiinflammatory agent. However, this formulation did not inhibit DOMS or muscle damage induced by eccentric exercise and also did not convincingly improve recovery after the DOMS-induced exercise. ${ }^{5}$

A herbal supplement containing Aphanizomenon flosaquae extract, berry extracts, nattokinase, serrapeptase, and curcumin, which have significant antioxidant and antiinflammatory properties, was used to facilitate postexercise recovery, but the herbal supplement was unable to accelerate recovery from upper arm eccentric exercise when compared with placebo. ${ }^{1}$ An innovative supplement produced from a blend of natural bioactive compounds containing polyphenols, which are extracted from the fruits of mangosteen, pomegranate, and elderberry, did reduce pain following eccentric exercise-induced damage and shortened muscle recovery. ${ }^{28}$ A great availability of antioxidant and antiinflammatory phytochemicals particularly rich in polyphenols, bilberry juice, suggested mild to moderate amplifications in exercise-induced DOMS. ${ }^{29}$ Lemon verbena extract commonly used in tea and various refreshing beverages, due to its significant antioxidant and anti-inflammatory actions, accelerated recovery after exhaustive exercise. ${ }^{6}$ Ginger supplementation was utilized to accelerate recovery of muscle power following powerful exercise. ${ }^{30}$ The protective role of oat protein was investigated against workout-induced muscle soreness, consequent inflammation, and loss of performance induced by downhill running, which considerably alleviated eccentric exercise-induced muscle soreness and inhibited the adverse effects of muscle strength. ${ }^{21}$ The majority of the studies agreed that the dietary supplements which have antioxidant and antiinflammatory properties provide minimum to no benefits for decreasing the severe indications linked with DOMS.

Curcumin supplementation effectively improved muscle function by attenuating the inflammatory response to eccentric exercise. Oral curcumin reduced pain connected with DOMS with the indication of moderate to large reduction in pain during exercise and a small reduction in the CK activity for enhanced recovery of muscle performance ${ }^{31}$ Our data collectively suggest that exercise induced muscle damage and inflammation during the study, and administering Cureit attenuated markers of muscle damage compared with the placebo. These results revealed that the employed exercise protocol was damaging muscles and Cureit attenuated the damage and decreased fluid accumulation. As a result of the bioavailable form of curcumin-Cureit has antioxidant, anti-inflammatory, and antirheumatic properties and these promising characteristics have contributed to the observed effects. ${ }^{10-15}$

In conclusion, the purpose of this study was to assess the efficacy of Cureit - a bioavailable form of curcumin could decrease damage from oxidative stress and inflammation related to acute muscle injury induced by eccentric continuous exercise. This study showed that oral ingestion of Cureit significantly reduced the DOMS, which is revealed by the attenuation of $\mathrm{CK}$ levels and increased $\mathrm{VO}_{2} \max$ value compared with placebo, and found safe for administration. Cureit has a positive effect on reducing muscle damage and it has advantageous effects on the recovery from eccentric exercise-induced muscle inflammation.

\section{ACKNOWLEDGMENTS}

The authors are grateful to the management of Plant Lipids (P) Ltd., Cochin, India, for support and inspiration. They express their gratitude to the laboratory members for their active help and cooperation.

\section{AUTHOR DISCLOSURE STATEMENT}

No competing financial interests exist.

\section{FUNDING INFORMATION}

This study was funded by Plant Lipids (P) Ltd., Cochin, India.

\section{REFERENCES}

1. Rynders CA, Weltman JY, Rynders SD: Effect of an herbal/ botanical supplement on recovery from delayed onset muscle soreness: A randomized placebo-controlled trial. J Int Soc Sports Nutr 2014;11:27.

2. Manimmanakorn N, Manimmanakorn A, Boobphachart D, Thuwakum W, Laupattarakasem W, Hamlin MJ: Effects of Zingiber cassumunar (Plai cream) in the treatment of delayed onset muscle soreness. J Integr Med 2016;14:114-120. 
3. Jenkins NDM, Housh TJ, Johnson GO, et al:: The effects of anatabine on non-invasive indicators of muscle damage: A randomized, double-blind, placebo-controlled, crossover study. J Int Soc Sports Nutr 2013;10:33.

4. Mickleborough TD, Sinex JA, Platt D, Chapman RF, Hirt M: The effects PCSO-524 ${ }^{\circledR}$, a patented marine oil lipid and omega-3 PUFA blend derived from the New Zealand green lipped mussel (Perna canaliculus), on indirect markers of muscle damage and inflammation after muscle damaging exercise in untrained men: A randomized, placebo controlled trial. J Int Soc Sports Nutr 2015;12:10.

5. Pumpa KL, Fallon KE, Bensoussan A, Papalia S: The effects of Lyprinol $\left({ }^{\circledR}\right)$ on delayed onset muscle soreness and muscle damage in well trained athletes: A double-blind randomised controlled trial. Complement Ther Med 2011;19:311-318.

6. Buchwald-Werner S, Naka I, Wilhelm M, Schütz E, Schoen C, Reule C: Effects of lemon verbena extract (Recoverben ${ }^{\circledR}$ ) supplementation on muscle strength and recovery after exhaustive exercise: A randomized, placebo-controlled trial. J Int Soc Sports Nutr 2018;15:5.

7. da Silva W, Machado ÁS, Souza MA, Mello-Carpes PB, Carpes FP: Effect of green tea extract supplementation on exerciseinduced delayed onset muscle soreness and muscular damage. Physiol Behav 2018;194:77-82.

8. Amalraj A, Pius A, Gopi S, Gopi S: Biological activities of curcuminoids, other biomolecules from turmeric and their derivatives-A review. J Tradit Complement Med 2016;7:205-233.

9. Amalraj A, Jude S, Varma K, et al.: Preparation of a novel bioavailable curcuminoid formulation (Cureit ${ }^{\mathrm{TM}}$ ) using PolarNonpolar-Sandwich (PNS) technology and its characterization and applications. Mater Sci Eng C Mater Biol Appl 2017;75: 359-367.

10. Gopi S, George R, Jude S, Sriraam VT: Cell culture study on the cytotoxic effects of "Cureit"-a novel bio available curcuminanti cancer effects. J Chem Pharm Res 2014;6:96-100.

11. Gopi S, George R, Sriraam VT: Cell culture study on the effect of bioavailable curcumin - 'Cureit" on elastase inhibition activity. Br Biomed Bull 2014;2:545-549.

12. Gopi S, George R, Sriraam VT: Anti oxidant potential of "Cureit"-A novel bioavailable curcumin formulation. Asian J Pharm Tech Innov 2014;2:123-127.

13. Gopi S, George R, Thomas M, Jude S: A pilot cross-over study to assess the human bioavailability of "Cureit" a bioavailable curcumin in complete natural matrix. Asian J Pharm Tech Innov 2015;3:92-96.

14. Gopi S, George R, Sriraam VT: Cell culture study on the effects of "cureit" hyaluronidase inhibition-Anti aging effects. Int $J$ Curr Res 2014;6:8473-8474.

15. Amalraj A, Varma K, Jacob J, et al.: A novel highly bioavailable curcumin formulation improves symptoms and diagnostic indicators in rheumatoid arthritis patients: A randomized, double-blind, placebo-controlled, two-dose, three-arm, and parallel-group study. J Med Food 2017;20:1022-1030.

16. Jude S, Amalraj A, Kunnumakkara AB, Divya C, Löffler B-M, Gopi S: Development of validated methods and quantification of curcuminoids and curcumin metabolites and their pharmacokinetic study of oral administration of complete natural turmeric formulation (Cureit ${ }^{\mathrm{TM}}$ ) in human plasma via UPLC/ESI-Q-TOFMS spectrometry. Molecules 2018;23:2415.
17. Jurenka JS: Anti-inflammatory properties of curcumin, a major constituent of Curcuma longa: A review of preclinical and clinical research. Altern Med Rev 2009;14:141-153.

18. Bisht K, Wagner KH, Bulmer AC: Curcumin, resveratrol and flavonoids as antiinflammatory, cyto- and DNA-protective dietary compounds. Toxicology 2010;278:88-100.

19. Nurenberg P, Giddings CJ, Stray-Gundersen J, Fleckenstein JL, Gonyea WJ, Peshock RM: MR imaging-guided muscle biopsy for correlation of increased signal intensity with ultrastructural change and delayed-onset muscle soreness after exercise. Radiology 1992;184:865-869.

20. Malm C, Sjodin TL, Sjoberg B, et al.: Leukocytes, cytokines, growth factors and hormones in human skeletal muscle and blood after uphill or downhill running. J Physiol 2004;556:9831000.

21. Gupta SC, Prasad S, Kim JH, et al:: Multitargeting by curcumin as revealed by molecular interaction studies. Nat Prod Rep 2011; 28:1937-1955.

22. Xia Z, Cholewa JM, Dardevet D, et al.: Effects of oat protein supplementation on skeletal muscle damage, inflammation and performance recovery following downhill running in untrained collegiate men. Food Funct 2018;9:4720-4729.

23. Gopi S, Jacob J, Varma K, et al:: Comparative oral absorption of curcumin in a natural turmeric matrix with two other curcumin formulations: An open-label parallel-arm study. Phytother Res 2017;31:1883-1891.

24. Udani JK, Singh BB, Singh VJ, Sandoval E: BounceBack ${ }^{\mathrm{TM}}$ capsules for reduction of DOMS after eccentric exercise: A randomized, double-blind, placebo-controlled, crossover pilot study. J Int Soc Sports Nutr 2009;6:14.

25. Jacob J, Amalraj A, Raj KKJ, Divya C, Kunnumakkara AB, Gopi $\mathrm{S}$ : A novel bioavailable hydrogenated curcuminoids formulation (CuroWhite ${ }^{\mathrm{TM}}$ ) improves symptoms and diagnostic indicators in rheumatoid arthritis patients-A randomized, double blind and placebo controlled study. J Tradit Complement Med 2019;9:346352.

26. Bannuru RR, Osani MC, Al-Eid F, Wang C: Efficacy of curcumin and Boswellia for knee osteoarthritis: Systematic review and meta-analysis. Semin Arthritis Rheum 2018;48:416-429.

27. Arent SM, Senso M, Golem DL, McKeever KH: The effects of theaflavin-enriched black tea extract on muscle soreness, oxidative stress, inflammation, and endocrine responses to acute anaerobic interval training: A randomized, double-blind, crossover study. J Int Soc Sports Nutr 2010;7:11.

28. Romain C, Freitas TT, Martínez-Noguera FJ, et al.: Supplementation with a polyphenol-rich extract, TensLess ${ }^{\circledR}$, attenuates delayed onset muscle soreness and improves muscle recovery from damages after eccentric exercise. Phytother Res 2017;31:1739-1746.

29. Lynn A, Garner S, Nelson N, Simper TN, Hall AC, Ranchordas MK: Effect of bilberry juice on indices of muscle damage and inflammation in runners completing a half-marathon: A randomised, placebo-controlled trial. J Int Soc Sports Nutr 2018; 15:22.

30. Matsumura MD, Zavorsky GS, Smoliga JM: The effects of preexercise ginger supplementation on muscle damage and delayed onset muscle soreness. Phytother Res 2015;29:887-893.

31. Nicol LM, Rowlands DS, Fazakerly R, Kellett J: Curcumin supplementation likely attenuates delayed onset muscle soreness (DOMS). Eur J Appl Physiol 2015;115:1769-1777. 